Document downloaded from:

http://hdl.handle.net/10251/35266

This paper must be cited as:

Masiá Vañó, J.; Eixerés Tomás, B.; Dols Ruiz, JF. (2011). Evaluation of the passive safety in cars adapted with steering control devices for disabled drivers. International Journal of Crashworthiness. 16(1):75-83. doi:10.1080/13588265.2010.514772.

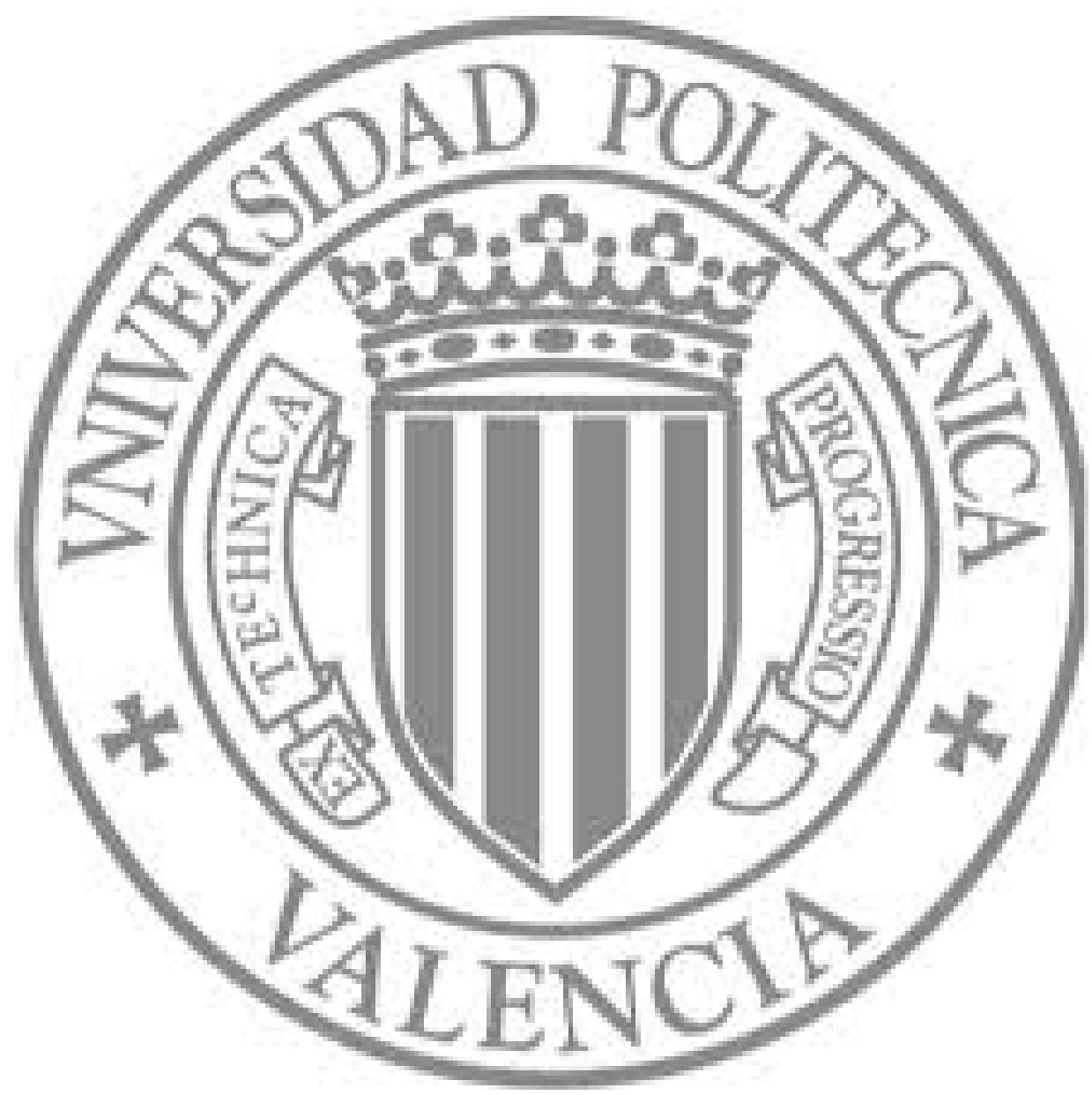

The final publication is available at

http://www.tandfonline.com/doi/abs/10.1080/13588265.2010.514772\#.Uo4LQ9Iz2Xw

Copyright Taylor \& Francis 


\title{
Evaluation of the passive safety in cars adapted with steering control devices for disabled drivers
}

\author{
J. Masiá*; B. Eixerés; J.F. Dols \\ Instituto de Diseño y Fabricación, Universidad Politécnica de Valencia, \\ Plaza Ferrándiz y Carbonell, 1. 03801 Alcoy (Alicante)
}

\begin{abstract}
The purpose of this research is to analyze the influence of steering control devices for disabled people on passive safety. It is based on the advances made in the modelling and simulation of the driver position and in the suit verification test. The influence of these devices is studied through airbag deployment and/or its influence on driver safety. We characterize the different adaptations that are used in adapted cars that can be found mounted in vehicles in order to generating models that are verified by experimental test. A three dimensional design software package was used to develop the model. The simulations were generated using a dynamic simulation program employing LSDYNA finite elements. This program plots the geometry and assigns materials. The airbag is shaped, meshed and folded just as it is mounted in current vehicles. The thermodynamic model of expansion of gases is assigned and the contact interfaces are defined. Static tests were carried out on deployment of the airbag to contrast with and to validate the computational models and to measure the behaviour of the airbag when there are steering adaptations mounted in the vehicle.
\end{abstract}

Keywords: Airbag; passive safety; disabled driver; steering control devices; finite elements.

\section{Introduction}

Nowadays, occupant safety is one of the principal objectives in the design of vehicles. Numerous innovations have appeared in recent years aimed at increasing safety in vehicles. The airbag is now one of the principal safety features installed to reduce injuries in the case of an accident, and is widely accepted as a means of protection for the occupants of a vehicle. Due to the important role they perform, the design and manufacture of the airbag, inflation techniques and opening mechanisms have been widely studied. However, research into the airbag FE model is minimal, and there appear to be no cases of research carried out on the introduction of steering device

\footnotetext{
* Jaime Masiá. Email: jmasia@mcm.upv.es
} 
adaptations into the survival space in case of an accident. Modern programs allow us to create a safety area which uses inflatable elements to reduce injury and fatal accidents caused by the impact of the head and upper body on the structures present in the vehicle. The introduction of steering assistance devices for disabled drivers is an additional element which needs to be studied within this area.

Computer models are particularly useful for creating improvements in safety when there is often little data available (Summers and Hollowell 2001). These models also allow us to increase the amount of information we have on airbag deployment in a wide range of conditions, information which would be difficult to obtain without these models. Some commercial FE analysis packages, such as LSDYNA, PAMCRASH and MADIMO, are potent tools for airbag models (Richert 2007). Airbag modeling through finite elements allows us to study the topology characteristics of the airbag and its contact with the occupant with precision and detail (Cheng et al. 2003).

In order to carry out computer analysis and ensure improvements to the safety system of a vehicle, it is critical in the computer model to integrate structural elements of the vehicle (steering wheel, steering device, etc.) which may intervene in the case of impact, with the passive safety devices (airbags, etc.). In the dynamics of airbag deployment, there are some important aspects to consider, such as how it is folded, and the airbag thermodynamic explosion model (Maklund and Nilsson 2002; Fokin et al. 2003; Haufe and Franz 2004).

In an ideal design configuration, the airbag system is optimized in such a way that, in the case of impact, the driver would make contact with the airbag only after it has completely inflated. At this instant, the airbag begins to deflate due to the holes in it 
and acts as a perfect cushion. If the contact takes place before this instant in which the airbag is not in the right position, and/or if there are other elements which prevent the correct deployment of the airbag, it will not perform its function correctly. Computer simulations are effective when researching these cases, as they provide the opportunity to carry out tests on a wide range of possible situations (Chawla et al. 2005).

During a frontal impact, if the driver is subjected by the seat belt, he is displaced forward and his head and thorax move suddenly forward and downward against the steering wheel. In this situation, a device assembled on the steering wheel (pins, tripins, concentric accelerators, systems of remote control, etc.) supposes an obstacle that can: cause the collision of the head against this, if there isn't any airbag, produce an interference in the airbag deployment that can decrease their efficacy or even project broken parts of these pieces causing damages to the occupants.

The research presented in this paper studies the variation produced on the passive safety if these devices are mounted. Some models of the survival space of the disabled driver have been developed with the purpose to simulate impact cases using several assistive steering devices. These models have been contrasted with the results of some "type" experimental tests. These models validated permit to reproduce the consequences that present the use of similar devices in conditions of similar impact without having to develop too many experimental tests.

\section{Definition of the problem}

The fundamental requirement of driving assistance devices is that they are designed to enable drivers with a wide range of disabilities to be able to drive. However, this fact must not mean a reduction in passive safety of the drivers of these vehicles. This 
research is aimed at determining the influence that these devices have on the safety area of the car. With this objective in mind, the work follows two lines: a simulation using finite element software aimed at achieving reliable models of behavior equivalent to that of real scenarios, along with another line involving experimental verification with which to contrast results from real experiments with simulation models (Khan and Moatamedi 2008, Crandall 1997). These models offer the opportunity to test multiple cases. It would not be viable to carry out such a variety of real tests.

Our principal objective is to verify whether driving assistance devices reduce passive safety in cars adapted for disabled drivers. This reduction in safety may be caused by different factors. Firstly, we checked if these devices have an effect on the deployment of the driver's airbag or if the devices cause breaks in or damage to them which may prevent them from carrying out their primary function. Secondly we studied the forces to which the adaptations are submitted and the possibility that if the devices are broken in some way, they may become extremely dangerous and cause serious injury. Finally, it is important to consider that the driver may be outside of the normal driving position due to the adaptations in the vehicle, and that as a consequence, driver safety may be compromised.

The main objective is to verify if the passive safety decrease with the assembled devices. This decrease in the passive safety can be caused by several situations:

1. The assembled devices interfere in the airbag deployment causing problems in the airbag unfolding in the interval of time required or producing damages in the bag. 
2. Due to the impact, the devices break and project pieces that can cause serious injuries.

3. At the instant of the impact, if the driver is "out of position", he could be injured impacting with these devices.

For this reason, we prepared a series of experimental tests using what are considered to be the most intrusive adaptations on the safety area of the car, and then we prepared their corresponding simulations in order to obtain reliable models which could then be used for further tests. From the adaptations chosen for the study, the following were considered to be fundamental: spinner knob, remote control knob, tri-pin and concentric accelerator (Figure 1).

Spinner knob is used to allow the driver to rotate the steering wheel with only one hand. Remote control knob is used to operate the lights, indicators, windscreen wipers, etc. without having to remove the hands from the steering wheel. Tri-pin is used by tetraplegics who have no use of one hand or wrist. The concentric accelerator is the driving device used by paraplegics.
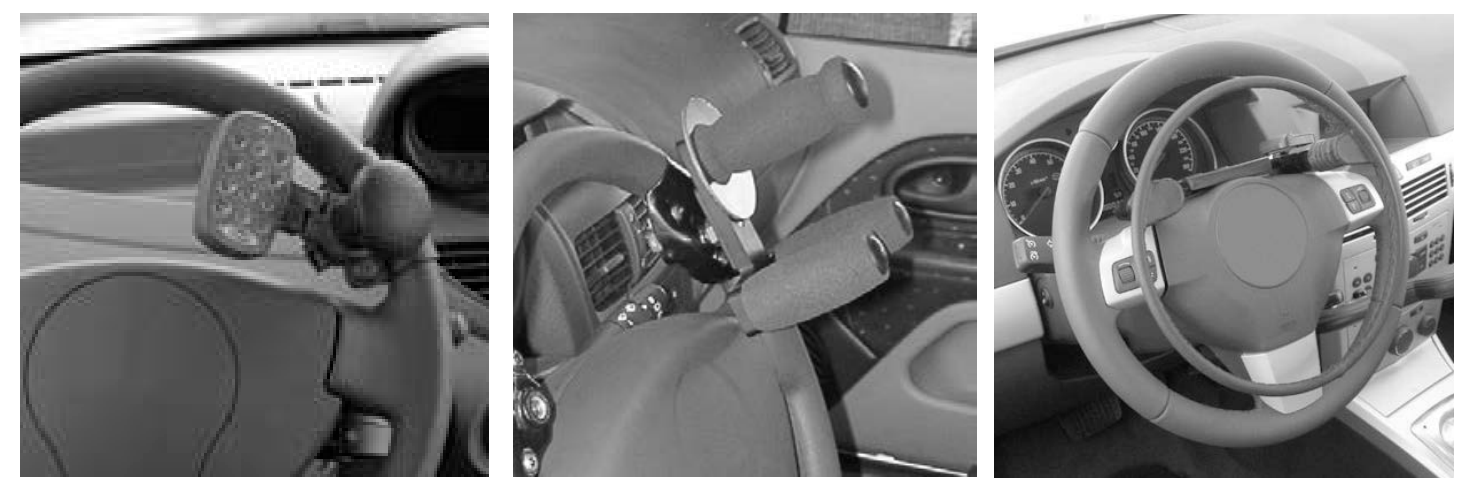

Figure 1. The adaptations which have the greatest influence on airbag deployment.

For the cases chosen, a series of tests were established in which the deployment of the airbag was analyzed from both the front and the side profiles for each of the 
adaptations. In these tests, we observed exactly how the airbag was deployed, and if this deployment was affected by the adaptations.

\section{Test equipment and methodology}

\subsection{Test Equipment}

The equipment used consisted of a test room suitably equipped with the safety measures necessary to test airbags of different vehicles. This room was fitted with a support structure onto which a steering wheel with airbag and the various assistance devices could be mounted. It was also appropriately lit and equipped with a high speed camera and a computer controlled camera firing system.

The support structure allowed us to place the steering wheel in the normal position within the vehicle. The wheel had a diameter of $340 \mathrm{~mm}$ and a $30 \mathrm{~mm}$ diameter rim. Onto this, we fitted the driver assistance devices in their various relevant positions. The airbag module contained the bag, the gas generator and the electric connector. The airbag is made of nylon, and the diameter both flat and deployed is $640 \mathrm{~mm}$. The bag has internal straps which connect to the airbag's rear support in such a way that the driver's face is protected at the moment that the airbag offers maximum protection.

The high speed camera used can store video at a maximum frequency of 1000 frames per second, at a resolution of 1024 by 1024 pixels, or can operate at a higher frequency with lower resolutions. The camera is controlled by computer and the entire process is synchronized to film the deployment of the airbag. The computer monitors the ambient conditions, presence detectors, light activation and advises when the camera begins filming, and then activates the airbag explosion if the conditions are 
right. The firing unit sends one or two pulses at specific times to film one or two phase airbags.

\subsection{Static airbag explosion test}

We planned a series of tests for different types of driving adaptations. From results of previous tests, we were able to select the devices which had most influence on airbag deployment. These were mounted frontally and for side profile in order to obtain sufficient information on the deployment of the airbag. The figure below shows the tri-pin and the concentric accelerator used in the tests.
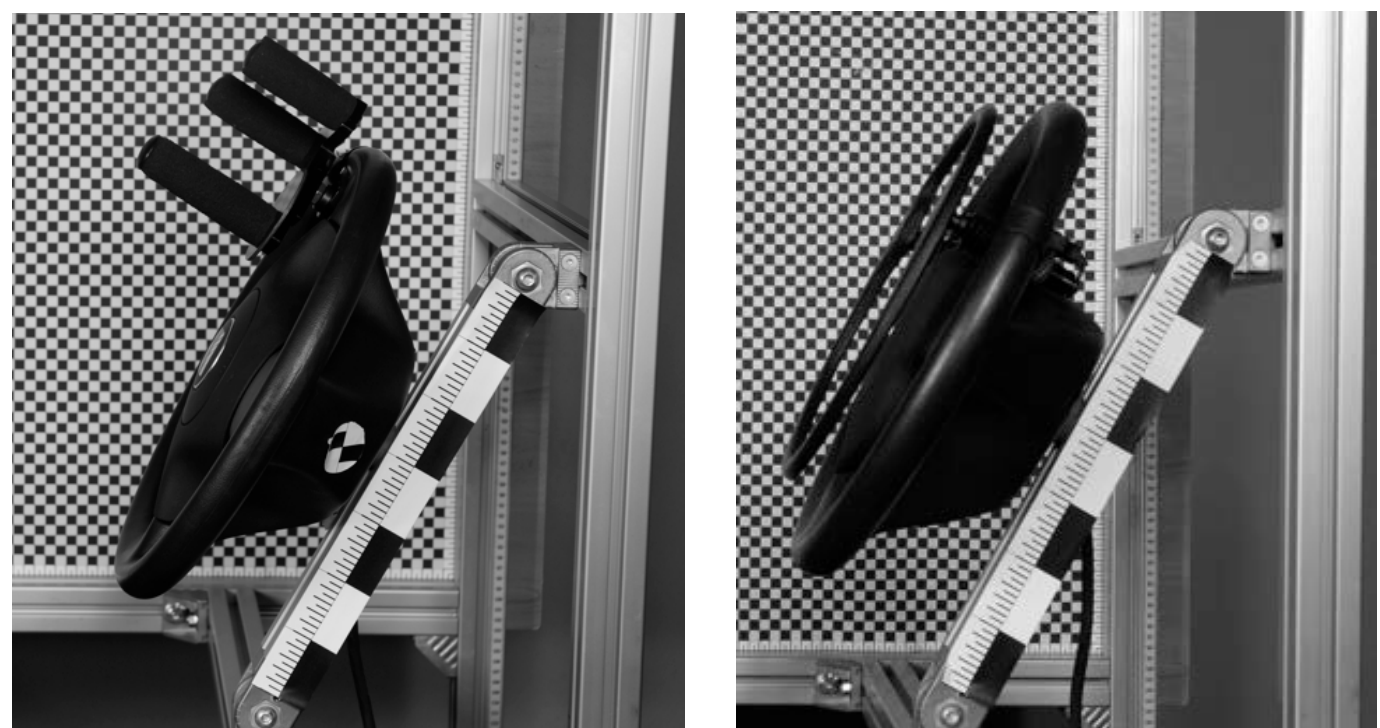

Figure 2. Example of the adaptations tested in the experiments.

With the aim of obtaining valid references for airbag deployment without driving assistance devices, we first carried out a test using a conventional airbag without mounting any devices on the steering wheel. In this way, we could establish the distance occupied by the airbag when deployed, and the different times taken for the airbag to be fully deployed. 
We used the plane of the steering wheel as a reference, and in the perpendicular direction in which the inflation of the airbag takes place, we measured the mid-line or outer edge of the airbag. This dimension is for use as a reference in later analyses, in which adaptations are mounted.

If there are no adaptations present, this outer edge of the airbag finishes parallel to the plane of the steering wheel and the distance is the same in any position. Once the adaptations are mounted, we measured the minimum and maximum distances with respect to the plane of the steering wheel in order to assess to what extent the airbag has deployed correctly.

\subsection{Test protocol}

We used a static test explosion procedure to record the deployment of the airbag mounted on the steering wheel and the interaction of the airbag with the adaptations. The specific steps in the procedure were as follows:

1) The steering wheel was positioned using the measurements previously established.

2) The control device was mounted in the position being tested.

3) The camera was positioned. The cameras and lighting were activated remotely from the control room. After the established delay, the airbag was activated using a 12 volt charge.

4) After the explosion of the airbag, we recorded the damage done to the steering wheel, the adaptations and the airbag. 
The data from each complete airbag deployment test was recorded by a high speed frontal camera. Then, the deployment of the airbag with the presence various adaptations was analyzed and compared with the reference test.

The area and the deployment of the airbag were measured with the camera in the side profile position. We used video analysis software which allowed us to deal with the image in each frame, converting the recorded image into the dimensions seen here (Dalrymple 1996).

\section{Simulation model}

The methodology employed to generate this simulation was made up of a first phase in which the three dimensional model of the driver's cockpit was developed. This was done with commercial three dimensional software. Once this first stage was completed, the model was applied to the finite element program in which the finite element mesh is generated. Finally, in the dynamic simulation software, the main conditioners of the model are specified, such as the materials and their characteristics, contact interfaces, gas expansion models, how the airbag is packed, etc (Bedewi et al. 1996).

Although we only analyzed the interaction between airbag and adaptation device, concerning the driving position, we considered the normal or standard driving position, in which the distance between driver and steering wheel (and therefore airbag) is the optimum position for correct functioning of the airbag. A disabled driver will often sit in a closer position to the steering wheel in order to better use the adaptations. 


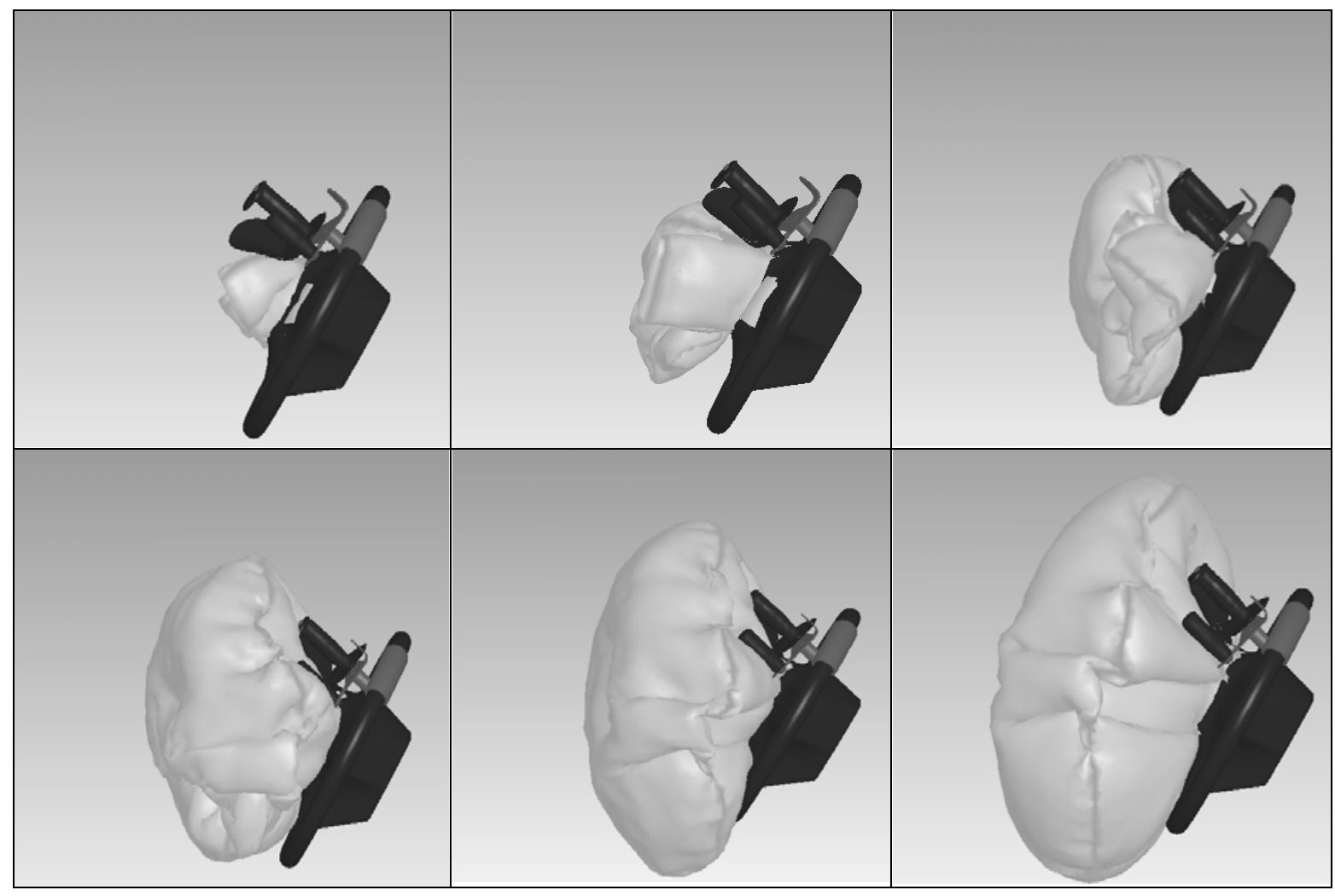

Figure 3. Example of an adapted steering wheel airbag explosion simulation.

\subsection{Airbag model}

ETA/VPG was used as a preprocessor to create the finite element model used in the study. There are several different types of airbag deployment model. Creating a FE model of a folded airbag is often a difficult task (Chawla et al. 2005). There are some tools currently available which automatically mesh and fold the airbag, however, in this project we first created the mesh of the deployed airbag, and then manually folded the airbag using ETA/VPG TM. In accordance with current folded airbag models, we did this in several steps. We first made a series of horizontal folds and then vertical folds so that the dimensions of the folded airbag are reduced to the size of the space available in the steering wheel. The figure below shows the FE model of a folded airbag before deployment. 


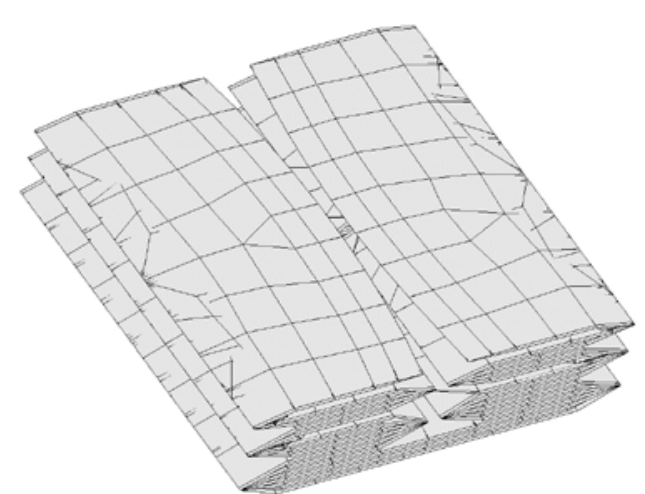

Figure 4. Folded airbag using FE software.

When using FE simulations of folded airbags, it is important to take certain factors into consideration. Algorithms used for folding tend to generate a fine mesh close to the fold in order to mark the exact line of the fold. In the model that we developed, we had to predefine a fold line when the mesh was generated so that the bag was correctly folded in the model. One of the most important factors to consider is that penetration between the layers must be avoided. Any internal penetration, although it may not be visible on the surface, has catastrophic consequences in the FE simulation of airbag deployment. The final point to consider is that the elements should have a minimum size to ensure stable simulations, which give explicit solutions and which are completed in an acceptable time

The FE airbag model was constructed for the simulation in LSDYNA ${ }^{\text {TM }}$ (LSTC 2007), where the thermodynamic behavior of the gas flow in the airbag includes hybrid effects from the detonator, defined by the keyword AIRBAG HYBRID. In this definition, many parameters or charge curves must be selected and defined. Of all of these, the flow ratio of the gas mass is one of the most important. In the figure below, the curve defines the temporal history of the gas mass flow ratio used. 


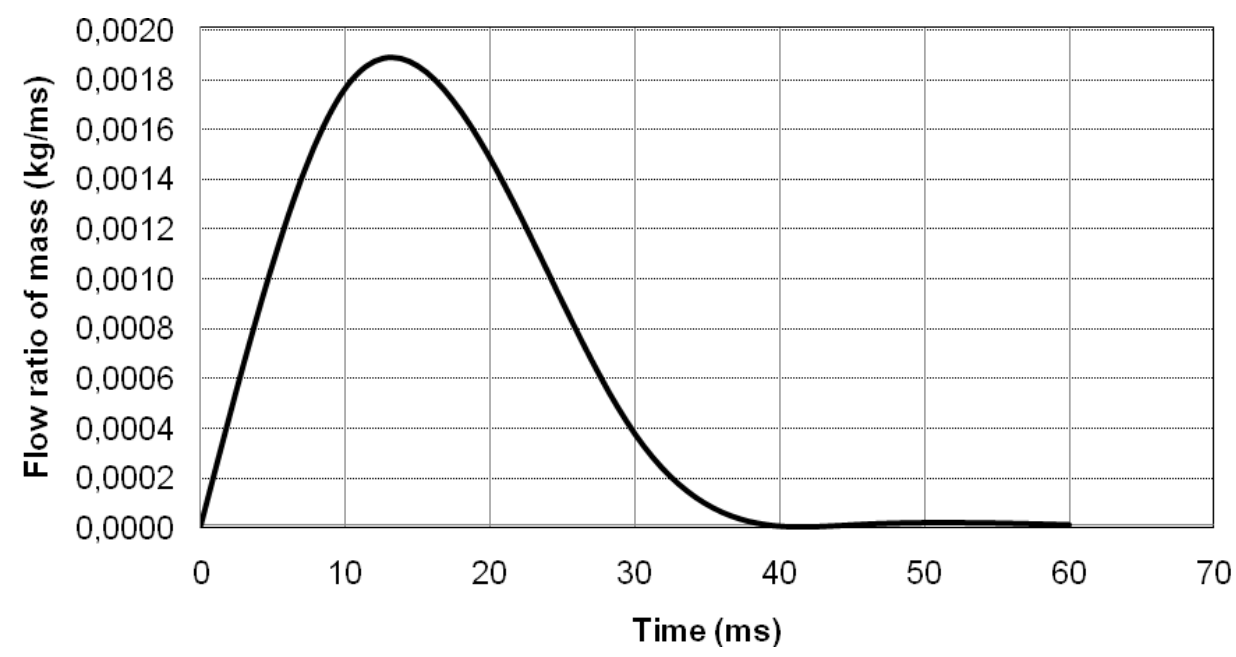

Figure 5. Flow ratio of mass entering the airbag.

The entry temperature of the gas also has an effect on how the airbag functions. In the cases studied, the entry temperature was assumed to be constant.

In this FE airbag model, we modeled four straps with the same elements as the skin of the airbag. With the aim of defining the area of the gas exit hole, we used a load curve which defines the exit area in function of absolute pressure. The data used in the model for the properties of the airbag textile material, the mass flow ratio and the gas entry temperature were provided by the manufacturers of the airbags and obtained from the relevant characterization tests. The contact between the skin of the airbag and the rest of the components which interact with it is defined by a contact type known as CONTACT_SURFACE_TO_SURFACE. The static rubbing coefficient and the dynamic rubbing coefficient between these parts must be small. We often observed large penetrations in the contact between the airbag skin and the rigid walls. We increased the scale factor for the problems of the sliding interface. 


\subsection{Airbag cover}

We proposed an approximation to the model in which we modeled the pre-established break line in the steering wheel for the airbag's escape. Generally, the join line in the cover is invisible from the outside. The plastic materials from which the cover is made, such as ABS o PP, weaken along this predetermined opening line due to tiny holes made by laser. This carrier part is covered by a layer which typically consists of a plastic, leather or textile sheet. This sheet accounts for the optical appearance of the interior and is usually supported by a thin layer of foam. It is impossible to model the laser holes with an FE mesh, because the mesh could contain elements that are too small, which would present numerical problems (time interval, etc.). For this reason, we studied other possibilities that would give more precise results:

The first alternative is to model using Shell plastic elements, which deform when subjected to force and fail when they reach the specified limit.

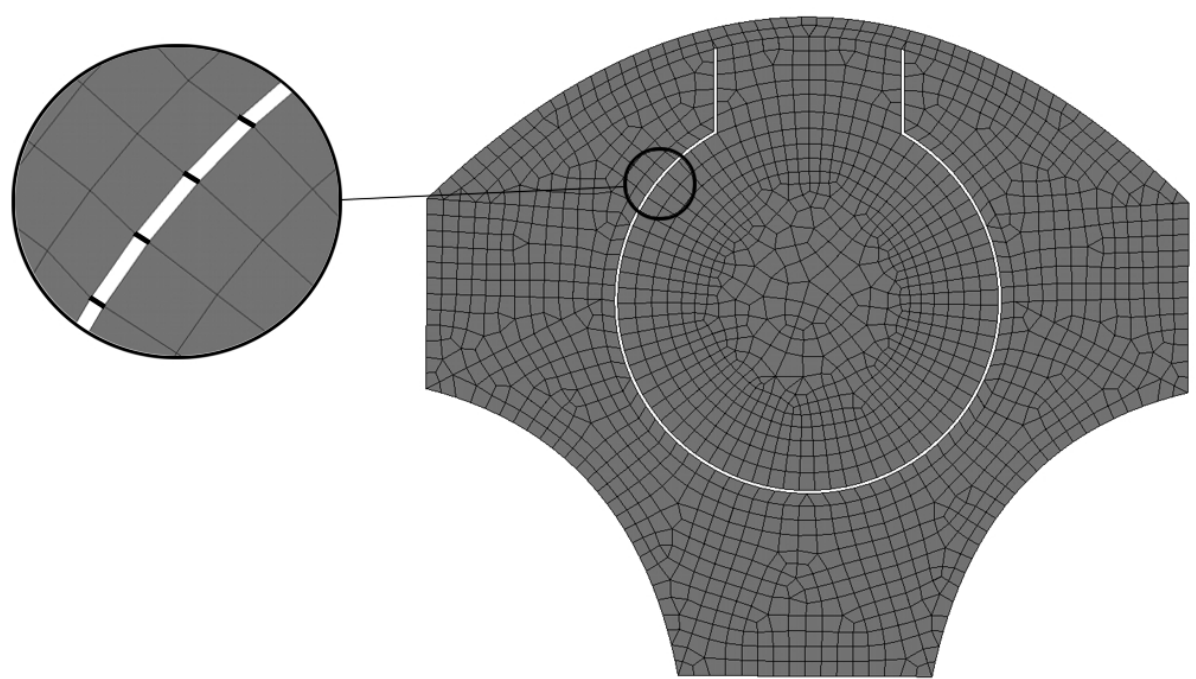

Figure 6. Model of the weld points on the airbag cover.

The second alternative is to model this joint using weld points, which are used to connect the opposite covers (see figure 6). A force is assigned to these beam elements. 
When the force limit is reached the beam element fails and is taken out of simulation by the solver. In this way, the cover can be moved freely at a local point where the weld point has failed.

In contrast, the experimental tests show that the second option is more appropriate to model these types of element (Ruff 2007), and so in the simulated model we used these.

The model of the carrier part was made in a 3D CAD design software. The geometry had a gap where the opening line was. When the mesh of the carrier part was made with shell elements, nodes at both parts of the opening line were joined with weld points. Some tests were done with so-called impactor tests. A specially prepared airbag cover part, fixed to the ground and positioned vertically, was penetrated with an accelerated impactor. Between the impactor and the force generator a force measure element was mounted. The opening force was calculated in this test. This force was used to assign the suit force to each weld point.

\subsection{Adaptations}

We carried out simulations using four different types of steering wheel adaptations: spinner knob, remote control knob, tri-pin and concentric accelerator. These devices are representative of the variety currently on the market, and according to previous work on the influence of these adaptations on passive safety (Dalrymple 1996; Pilkey et al. 1996), they are among those that interfere most with the correct working of the front airbag, as they are fixed to the steering wheel using a sleeve and are mounted toward the inside of the steering wheel. As can be seen in the figure, they can be fixed 
in any position on the steering wheel, depending on the personal requirements and comfort of the driver.
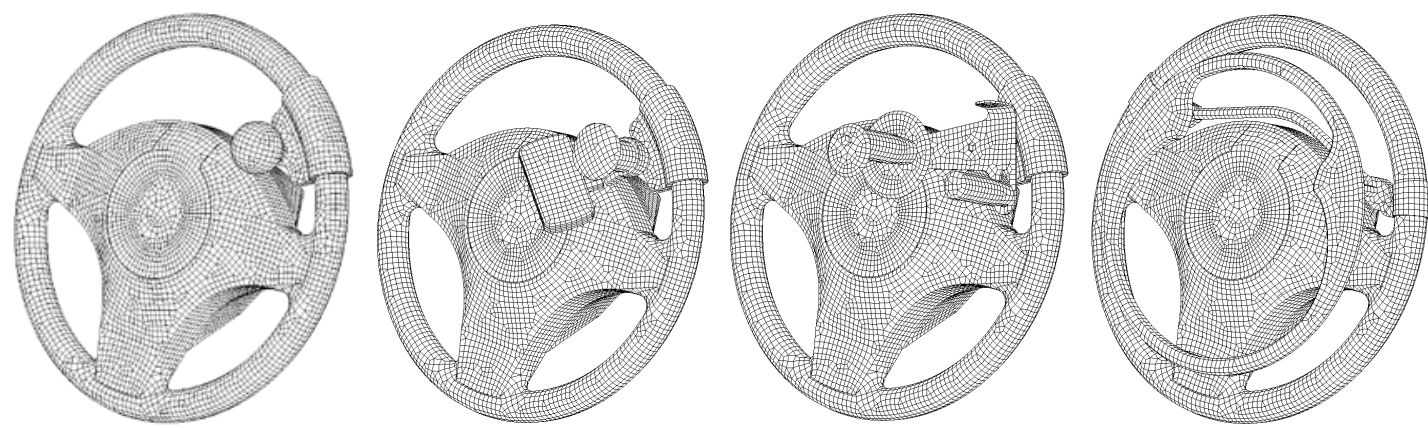

Figure 7. Steering wheel adaptations used in the study.

\section{Results}

The reference tests carried out allowed us to validate the models developed using finite elements, allowing us to carry out a great number of different configurations which would have been impossible using real experiments. Figure 8 shows comparative images of the experimental test and the simulation.
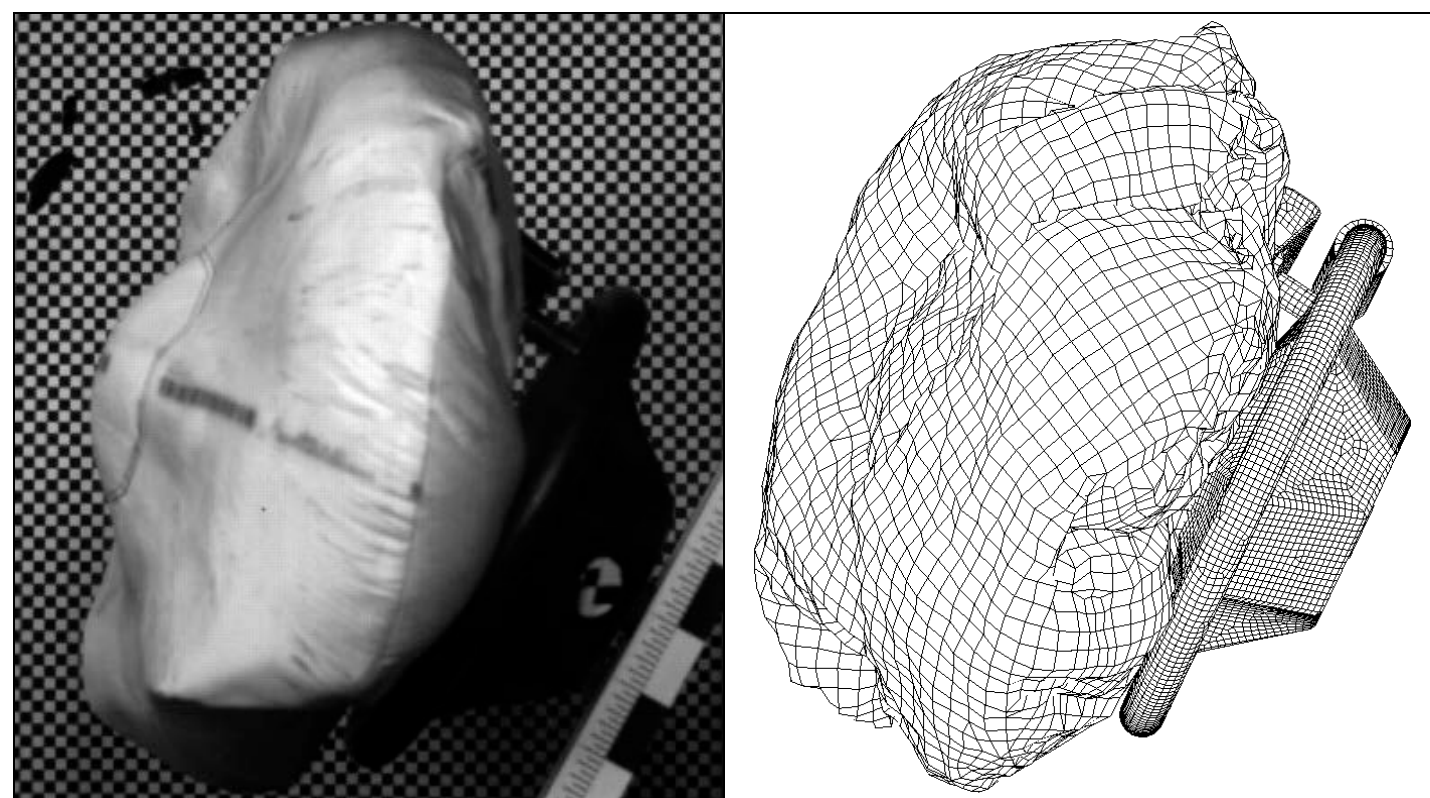

Figure 8. Verification of the FE model - experimental airbag. 
Part of the data used in simulations to determine the influence of adaptations on airbag deployment is a representation of the tensions present. In this we can see where the greatest tensions are produced by the explosion, and it also allows us to see if the adaptation fails under these tensions. If this should happen, driver safety could be greatly reduced by the presence of sharp edges resulting from broken pieces.

Spinner knob is relatively small compared with the other devices and has less effect on driver safety. It is larger, and when it is in certain positions, it has a considerable affect on the airbag's correct deployment. Moreover, if the driver's hands are in the deployment area, the injuries caused are considerable.

Tri-pin has even greater dimensions, as well as a design that has a greater affect on correct airbag deployment. In this case, we saw problems such as shattering or breaking in the device, as well as deformation of the airbag (Fig. 8).
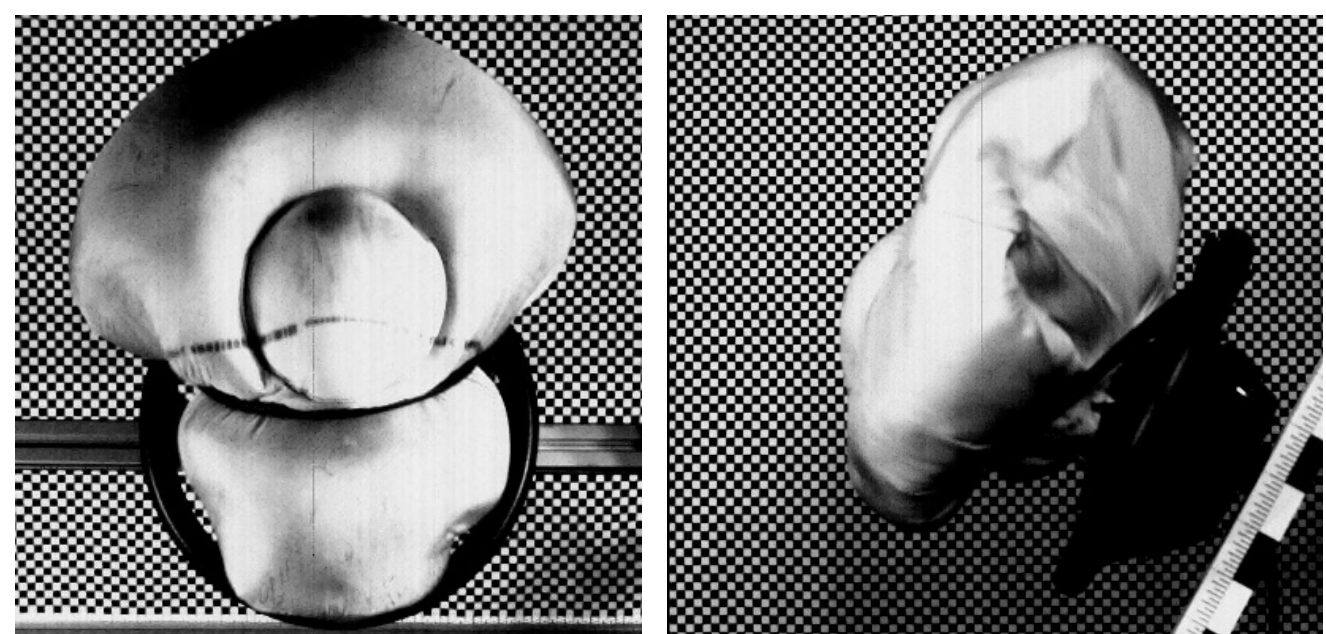

Figure 9. Problems caused in airbag deployment by the concentric accelerator.

In certain cases, when its geometry is relatively small with respect to the circumference of the steering wheel, the concentric accelerator produces breaks in the airbag. We also observed cases where the device blocked the airbag, preventing its correct deployment. 


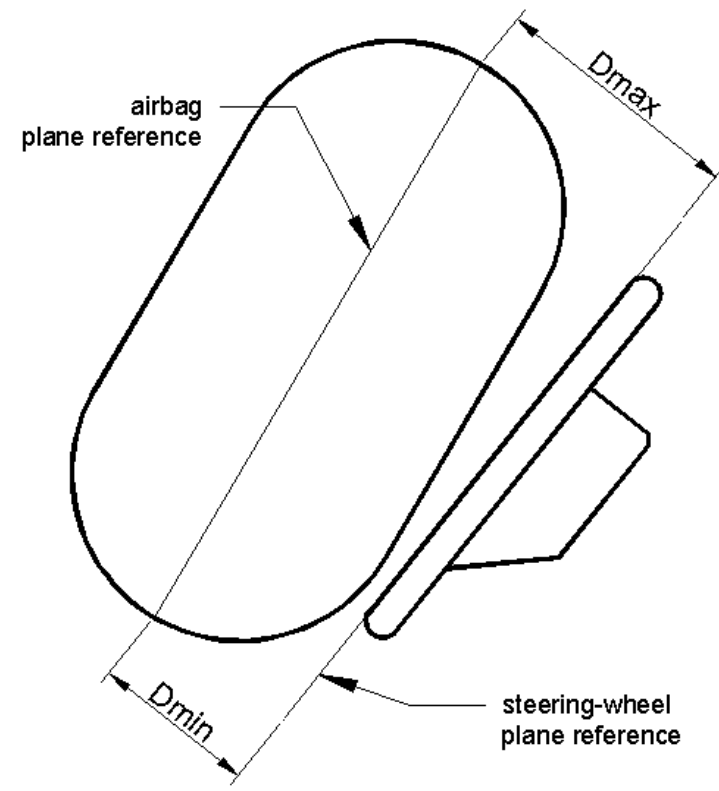

Figure 10. References used to measure maximum and minimum distances.

The parameters used to evaluate influence in passive safety of adaptations are resumed in Table 1 for studied devices. Maximum and minimum distances are used to verify the position of the airbag (Figure 10). The volume and the bag deformation (shape) are useful to know the success airbag deployment. These parameters are measured $40 \mathrm{~ms}$ after the frontal impact. In this interval of time the airbag should be perfectly deployed and the driver will be protected to impact against the steering wheel and the adaptations.

\begin{tabular}{|l|l|l|l|l|}
\hline Adaptation shape & $\begin{array}{l}\text { Dmin } \\
(\mathrm{mm})\end{array}$ & $\begin{array}{l}\text { Dmáx } \\
(\mathrm{mm})\end{array}$ & $\begin{array}{l}\text { Volume } \\
\text { average }\left(\mathrm{dm}^{3}\right)\end{array}$ & Bag shape \\
\hline No adaptation & $120-124$ & $129-134$ & 41.5 & No deformation \\
\hline Spinner knob & $118-122$ & $130-136$ & 40.3 & Little deformation \\
\hline Remote control knob & $111-119$ & $122-135$ & 39.6 & Little deformation \\
\hline Tri-pin & $87-91$ & $143-151$ & 37.9 & Medium deformation \\
\hline Concentric accelerator & $3-132$ & $112-171$ & 36.1 & Large deformation \\
\hline
\end{tabular}

Table 1. Parameters used to evaluate influence in passive safety of adaptations 


\section{Conclusions}

The results obtained from these tests lead us to draw various conclusions:

1) Devices which are mounted over the airbag must not be used. Devices which are struck by the airbag when it opens must not be used. Adaptations such as the concentric accelerator often cover the area of airbag deployment and therefore are not recommended. The experimental tests and simulations have both shown that they can seriously interfere in the correct deployment of the airbag. So that the driver is not affected by the mounting of these adaptations. The driver should be seated in such a position that they do not strike the adaptations before the airbag is deployed.

2) From the data obtained in this study, we can deduce that tri-pin devices reduce the possibility that the airbag deploys correctly and in the correct time and can cause injury to the hand in the explosion process. In the case that the risk is considered to be great, it is advisable to deactivate the airbag.

3) Knob has little affected on the static deployment of the airbag. Therefore adaptations of this type are preferable to those of two or three points, as they have both less effect on the airbag and on possible hand injuries.

4) The fact that none of the adaptations perforate or pierce the airbag leads us to believe that in the case of impact, the bag leaves enough space between the adaptation and the driver to prevent injury through driver impact on the adaptation. 
We have generated reliable models with which to contrast results from real experiments. These models allow us to analyze the passive safety of disabled drivers. From the study, we can conclude that the interference of driving adaptations in the airbag deployment space has considerable effect on the correct positioning of the airbag at the moment of impact, creating a considerable reduction in the passive safety of disabled drivers. The adaptations which interfere to a greater degree in the deployment area of the airbag are those which produce incorrect positioning of the airbag, as is the case of the remote control knob and tri-pin.

\section{REFERENCES}

Bedewi NE, Marzougui D, Motevalli V. 1996. Evaluation of Parameters Affecting Simulation of Airbag Deployment and Interaction with Occupants. International Journal of Crashworthiness. 1(4): 339-354.

Chawla A, Mukherjee S, Sharma A. 2005. Development of FE meshes for folded airbags. International Journal of Crashworthiness. 10(3): 259-266.

Cheng Z, Rizer AL, Pelletiere JA. 2003. Modeling and simulation of OOP occupant-airbag interaction SAE Technical Paper Series. 510.

Crandall JR, Bass CR, Pilkey WD, Morgan RH, Eppinger RH, Miller HJ, Sikorski J. Thoracic Response and Injury with Belt, Driver Side Airbag, and Constant Force Retractor Restraints, International Journal of Crashworthiness, vol. 2, pp. 119-132, 1997.

Dalrymple G. 1996. Effects of assistive steering devices on air bag deployment. SAE Technical Paper Series. 1139.

Fokin D, Lokhande N, Fredriksson L. 2003. On airbag simulation in LS-DYNA with the use of the arbitrary Lagrangian Eulerian method. Paper presented at the 4th European LS-Dyna Conference, Ulm, Germany.

Haufe A, Franz U. 2004. On the simulation of out-of-position load cases with the ALE-method Airbag. Paper presented at the 7th International Symposium and Exhibition on Sophisticated Car Occupant Safety Systems, Karlsruhe, Germany.

Khan MU, Moatamedi M. 2008. A review of airbag test and analysis. International Journal of Crashworthiness. 13(2): 67-76.

Khan MU, Moatamedi M, Zeguer T, Souli M. 2008. Multiphysics out of position airbag simulation. International Journal of Crashworthiness. 13(2): 159-166

Marklund PO, Nilsson L. 2002. Simulation of airbag deployment using a coupled fluid-structure approach. Paper presented at the 7th International LS-Dyna Conference, Detroit, USA.

Livermore Software Technology Corporation (LSTC). 2007. LS-DYNA Keywords user’s manual. 
Pilkey WD, Thacker J, Shaw G. 1996. Airbag interaction with and injury potential from common steering control devices. Final Project Report DOT-HS-808-580. National Highway Safety Administration. Washington, D.C. USA.

Richert J, Coutellier D, Götz C, Eberle W. 2007. Advanced smart airbags: The solution for real-life safety. International Journal of Crashworthiness. 12(2): 159-171.

Ruff C, Jost T, Eichberger A, 2007. Simulation of an airbag deployment in out-of-position situations. Vehicle System Dynamics. 45(10): 953-967.

Summers SM, Hollowell WT. 2001. Crashworthiness modeling activities NHTSA's. Proceedings of the Seventeenth International Conference on Enhanced Safety of Vehicles. 251. 